\title{
Intravitreal aflibercept for the treatment of choroidal neovascularization associated with pathologic myopia: a pilot study
}

This article was published in the following Dove Press journal:

Clinical Ophthalmology

4 November 2016

Number of times this article has been viewed

\section{Andrii R Korol \\ Oleg S Zadorozhnyy Volodymyr O Naumenko Taras B Kustryn Nataliya $\vee$ Pasyechnikova}

The Filatov Institute of Eye Diseases and Tissue Therapy of the National Academy of Medical Sciences of Ukraine, Odessa, Ukraine
Correspondence: Andrii R Korol

The Filatov Institute of Eye Diseases and Tissue Therapy of the National Academy of Medical Sciences of Ukraine, 49/5 I Frantsuzkii Blvd, Odessa 6506I, Ukraine Tel +380 43287298352

Fax +380 43287760435 Email laserfilatova@gmail.com
Purpose: To determine the efficacy of intravitreal aflibercept injections for the treatment of patients with choroidal neovascularization (CNV) associated with pathologic myopia.

Methods: In this uncontrolled, prospective cohort study, 31 eyes of 30 consecutive patients affected by $\mathrm{CNV}$ associated with pathologic myopia were treated with intravitreal aflibercept $(2 \mathrm{mg}$ ) as needed following two initial monthly doses and observed over a 12-month follow-up period. The primary endpoint was change in best-corrected visual acuity (BCVA) at month 12 , while central retinal thickness (CRT) on optical coherence tomography (OCT), neovascularization activity on fluorescein angiography, the number of aflibercept injections administered, and safety were examined as secondary endpoints.

Results: Patients received a mean of 2.6 intravitreal aflibercept injections over the 12-month study period. Compared with baseline, BCVA improved significantly at all time points $(P<0.05)$. Mean (standard deviation [SD]) decimal BCVA was $0.2(0.1)$ at baseline and $0.35(0.16)$ at month 12. The greatest improvement in BCVA was seen within the first 2 months $(P=0.01)$. Mean (SD) CRT on OCT decreased from 285 (62) $\mu \mathrm{m}$ at baseline to 227 (42) $\mu \mathrm{m}(P=0.01)$ at month 12. There was a continuous decrease in mean CRT on OCT over time. No cases of endophthalmitis, uveitis, stroke, or retinal detachment were noted. No patient demonstrated an intraocular pressure $>20 \mathrm{mmHg}$ during any study visit.

Conclusion: The 12-month results of intravitreal aflibercept for myopic CNV using an as-needed regimen were positive, showing benefits in visual and anatomic outcomes and an acceptable tolerability profile.

Keywords: aflibercept, angiogenesis inhibitors, choroidal neovascularization, eye diseases, pathologic myopia

\section{Introduction}

A recent systematic review has indicated that the prevalence of pathologic myopia (PM) is $1 \%-3 \%$ in adults, and that $5 \%-11 \%$ of patients with PM develop choroidal neovascularization (CNV). ${ }^{1}$ Several of the phenotypic features of PM are associated with an increased risk of myopic CNV; these include lacquer cracks, patchy atrophy, thinning of the choriocapillaris and choroid, ${ }^{2,3}$ and CNV in the fellow eye. ${ }^{4}$ Furthermore, in 2003, Ohno-Matsui et al showed that after the initial presentation of myopic CNV, CNV develops in the fellow eye in $35 \%$ of patients within 8 years. ${ }^{2}$

Because of the poor natural history of myopic CNV, different treatment approaches have been investigated. Thermal laser photocoagulation, transpupillary thermotherapy (TTT), and photodynamic therapy (PDT) with verteporfin have shown some benefits, but long-term efficacy and safety outcomes are disappointing. ${ }^{5-8}$ 
Anti-vascular endothelial growth factor (anti-VEGF) agents such as bevacizumab, ranibizumab, and aflibercept are widely used for the treatment of many retinal diseases. Bevacizumab is a recombinant humanized monoclonal antibody that binds all isoforms of VEGF-A, ${ }^{9}$ while ranibizumab is an affinity-matured antigen-binding fragment derived from bevacizumab. ${ }^{9}$ Aflibercept is a soluble decoy receptor fusion protein that binds VEGF-A, VEGF-B, and placental growth factor. ${ }^{9,10}$ Randomized clinical trials have demonstrated the efficacy of ranibizumab and aflibercept in wet age-related macular degeneration, ${ }^{11-13}$ macular edema secondary to central retinal vein occlusion, ${ }^{14-17}$ and diabetic macular edema. ${ }^{18,19}$ Intravitreal ranibizumab and aflibercept are generally well tolerated in patients of different ages with different retinal diseases, and these drugs have similar safety profiles. ${ }^{11-19}$

Based on data from a prospective open-label phase 2 trial ${ }^{20,21}$ as well as the randomized controlled phase 3 RADIANCE trial, ${ }^{22}$ ranibizumab was recently approved in the European Union and Japan for the treatment of visual impairment due to CNV secondary to PM. MYRROR, a phase 3 study, evaluates the efficacy and safety of intravitreal aflibercept in eyes with CNV due to PM. Interim study results were presented in 2013 by Ohno-Matsui et al, and showed greater visual and anatomic improvements in eyes treated with intravitreal aflibercept compared with sham. ${ }^{23}$

The purpose of the current pilot study was to investigate the efficacy of intravitreal injection of aflibercept in patients with CNV associated with PM.

\section{Patients and methods Protocol}

Consecutive patients who presented to the Laser Department of the State Institution "The Filatov Institute of Eye Diseases and Tissue Therapy of the NAMS of Ukraine" with signs and symptoms consistent with CNV associated with PM were examined and screened for entering the study. Inclusion criteria were high myopia (defined as myopia of $\geq-6.00 \mathrm{D}$ and/or axial length of $\geq 26.5 \mathrm{~mm}$ ), new onset of CNV associated with $\mathrm{PM}<2$ months, age $\geq 18$ years, myopic retinopathy (one or more of the following signs: staphyloma, lacquer cracks, Fuchs spot, and myopic chorioretinal atrophy), and absence of inflammation at screening. Ocular exclusion criteria included any other cause of $\mathrm{CNV}$, intraocular or periocular inflammation, known glaucoma or clinical suspicion of glaucoma on presentation, ocular hypertension (intraocular pressure $[\mathrm{IOP}]>24 \mathrm{mmHg}$ ), or fundus observation complicated by opacity of optic media. Nonocular exclusion criteria included pregnancy, lactation, and inability to provide consent. Eligible subjects were given a detailed explanation concerning their disease and the proposed study and treatment. They were then provided the opportunity to consider study participation, and only then, their signed and informed consent was obtained.

This study was approved by the State Institution "The Filatov Institute of Eye Diseases and Tissue Therapy of the NAMS of Ukraine" Research Ethics Board before it was commenced. All procedures were performed in accordance with the 1964 Declaration of Helsinki and Good Clinical Practice guidelines.

The study was conducted between March 2013 and September 2014. Patient enrollment took place from March 2013 to September 2013.

\section{Intervention}

Two obligatory loading injections of intravitreal aflibercept were administered at baseline and at month 1 . Subsequent injections were given on an as-needed (PRN) basis. Retreatment criteria included persistence of $\mathrm{CNV}$ or macular edema or recurrence of $\mathrm{CNV}$ or macular edema (increase in retinal thickness in the foveal area $>50 \mu \mathrm{m}$ ), sub- or intraretinal fluid and/or intraretinal cysts on optical coherence tomography (OCT), or subretinal hemorrhage observed during dilated fundus examination.

\section{Study visits}

All enrolled patients underwent baseline ophthalmologic examination and investigations including measurements of best-corrected visual acuity (BCVA) using the Shevalev chart (a Soviet analog to the Early Treatment of Diabetic Retinopathy Study chart); IOP measurements; pupillary, biomicroscopic, and dilated fundus examination; as well as spectral domain OCT (Spectralis; Heidelberg Engineering, Heidelberg, Germany) of the macular area, color fundus photography, and fluorescein angiography (FA) (TRC-50EX; Topcon, Tokyo, Japan).

The area of hyperfluorescence was measured in the early phase of FA images using MultiSpec computer software. Hyperfluorescence before and after treatment was compared and was described as CNV closure (absence of hyperfluorescence) or CNV persistence (presence of hyperfluorescence). Staining from fibrosis was differentiated from CNV persistence by late hyperfluorescence resulting from the accumulation of fluorescein dye into the subretinal scar.

Subsequently, all patients were reexamined monthly for 12 months. Follow-up examinations involved ophthalmologic 
examination including BCVA measurements, IOP measurements, and dilated fundus examination. OCT and color fundus photography were also repeated at each of these visits. FA was performed in the case of decrease in BCVA and/or increase of macular edema or suspicion of CNV recurrence.

In addition to all study visits, a telephone safety check was performed 1 day after each intervention to monitor for complications related to the intraocular injection. Specifically, the following adverse events were ruled out: elevated IOP, endophthalmitis, retinal tear, retinal detachment, vitreous hemorrhage, uveitis, and corneal abrasion. Any other complications encountered were also noted.

\section{Outcome measures}

The primary outcome was the change in BCVA from baseline to month 12 . The secondary outcomes included change in central retinal thickness (CRT) in the foveal area on OCT from baseline to month 12 , neovascularization activity on FA, the number of aflibercept injections administered, and safety.

\section{Analysis}

Using a last observation carried forward strategy for the replacement of missing data, baseline and final outcome measurements for the primary and secondary outcome variables were compared utilizing a repeated measures analysis of variance design with one between-subjects factor. The independent variable of group assignment was included for treatment effect. Any statistically significant baseline differences between the groups were entered as covariates in the model.

Baseline characteristics were compared with Student's $t$-test, $\chi^{2}$, or Fisher's exact test, as appropriate.

\section{Results}

Thirty patients ( 31 eyes) completed the 12-month follow-up examination. The mean (standard deviation [SD]) age of patients was 43.0 (12.4) years, and 25 (83.0\%) were female. Seven $(23 \%)$ patients had a past medical history of arterial hypertension and two $(6.7 \%)$ were smokers. All patients had a high myopia with myopic retinopathy (staphyloma, lacquer cracks, Fuchs spot, and myopic chorioretinal atrophy). All patients presented with a predominantly classic CNV in the macular area. A total of seven (23\%) cases of CNV were juxtafoveal and 24 (77\%) were subfoveal. Mean (SD) baseline decimal BCVA was $0.2(0.1)$. Mean (SD) baseline retinal thickness in the foveal area was 285 (61.6) $\mu \mathrm{m}$.

\section{Primary and secondary outcomes}

Compared with baseline, BCVA improved significantly at all time points $(P<0.05)$. Mean BCVA improved from 0.2 $(0.1)$ at baseline to $0.35(0.16)$ at month 12 (Figure 1). The greatest improvement in BCVA was seen within the first 2 months $(P=0.01)$. Visual results over time are shown in Table 1. At 1 year, an improvement in BCVA of $\geq 3$ lines was seen in five (16\%) eyes. Fourteen (45\%) eyes improved by $\geq 2$ but $<3$ lines, and eight ( $26 \%$ ) eyes improved by $\geq 1$ but $<2$ lines. BCVA was unchanged in four (13\%) eyes. None of the treated eyes lost $\geq 1$ line of vision.

Mean (SD) CRT on OCT showed a reduction from 285.0 (62) $\mu \mathrm{m}$ at baseline to $227.0(42) \mu \mathrm{m}(P=0.01)$ at month 12 (Figure 2). There was a continuous decrease in mean CRT on OCT over time (Table 1).

Mean (SD) area of hyperfluorescence decreased from $0.41(0.38) \mathrm{mm}^{2}$ at baseline to $0.32(0.22) \mathrm{mm}^{2}$ at 12 months $(P=0.04)$.

All patients received two obligatory loading injections at baseline and at month 1 . The mean (SD) number of injections was $2.2(0.5)$ and $2.6(0.9)$ at months 3 and 12, respectively. A total of 18 patients (18 eyes) received only two loading injections and did not require additional injections. The maximum number of injections (5.0) was administered to three patients (three eyes).

Three patients (three eyes) showed recurrence of CNV, which was successfully treated with additional injections. Each eye had one recurrence of CNV. No case of persistent $\mathrm{CNV}$ was noted during 12 months of follow-up.

Figure 3 provides representative examples of anatomic improvements following intravitreal aflibercept injections. As seen in the fundus photographs (Figure $3 \mathrm{~A}$ and $\mathrm{B}$ ), this patient experienced regression in CNV lesion size, reduction of angiographic leakage on FA (Figure 3C and D), and decrease in CRT (Figure 3E and F) after two intravitreal injections.

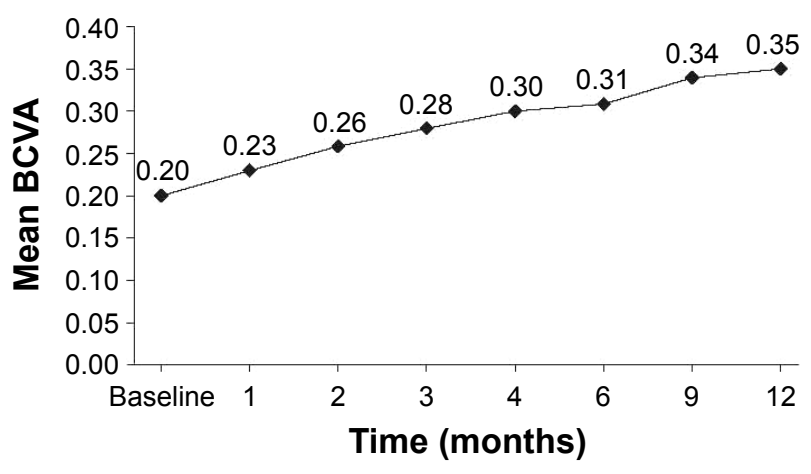

Figure I Change in mean BCVA following treatment with intravitreal aflibercept for myopic choroidal neovascularization.

Abbreviation: BCVA, best-corrected visual acuity 
Table I Visual and anatomical results in patients treated with CNV associated with pathologic myopia

\begin{tabular}{|c|c|c|c|c|c|}
\hline Results & Baseline $(n=3 I)$ & Month I $(n=3$ I) & Month $3(n=3 I)$ & Month $6(n=31)$ & Month I $2(n=3$ I) \\
\hline Mean (SD) decimal BCVA & $0.2(0.1)$ & $0.23(0.1)$ & $0.26(0.14)$ & $0.31(0.15)$ & $0.35(0.16)$ \\
\hline$P$-value & & 0.03 & 0.001 & 0.001 & 0.001 \\
\hline Mean (SD) CRT, $\mu \mathrm{m}$ & $285.0(62)$ & $269.0(53)$ & $246.0(47)$ & $236.0(45)$ & $227.0(42)$ \\
\hline$P$-value & & 0.01 & 0.001 & 0.003 & 0.01 \\
\hline CNV closure rate, no (\%) & & & $|6 / 3|(52)$ & $24 / 31$ (77) & $30 / 31$ (97) \\
\hline Mean (SD) injections & & & & & $2.6(1.0)$ \\
\hline
\end{tabular}

Abbreviations: BCVA, best-corrected visual acuity; CNV, choroidal neovascularization; CRT, central retinal thickness; SD, standard deviation.

\section{Complications}

Side effects observed after intravitreal injections included eye pain ( $8 / 81$ injections, $10 \%$ ), subconjunctival hemorrhage (6/81 injections, 7\%), and vitreous reflux (14/81 injections, $17 \%)$. No cases of endophthalmitis or of uveitis, stroke, or retinal detachment were noted. No patient demonstrated an IOP $>20 \mathrm{mmHg}$ during any study visit.

\section{Discussion}

Before the introduction of anti-VEGF therapy, laser photocoagulation, TTT, PDT with verteporfin, and different surgical techniques were used for the treatment of myopic $\mathrm{CNV}$; however, these treatment approaches were typically unsatisfactory in that they failed to improve visual acuity to a clinically relevant extent in the mid to long term.

Laser photocoagulation of well-defined subfoveal $\mathrm{CNV}$ secondary to age-related macular degeneration has been described as successful in stopping CNV activity, but the ensuing damage to the overlying neurosensory retina compromised visual acuity. ${ }^{24,25}$ In myopic patients, laser photocoagulation of extrafoveal CNV has poor long-term results because of atrophic progression even in eyes free of CNV recurrence. ${ }^{8,26,27}$ In a comparison of laser photocoagulation with the natural history of myopic $\mathrm{CNV}$, lasertreated eyes had statistically better BCVA at 2 years, but this difference was insignificant after 5 years. ${ }^{24,26}$ In a study of eyes with extrafoveal CNV secondary to PM and treated

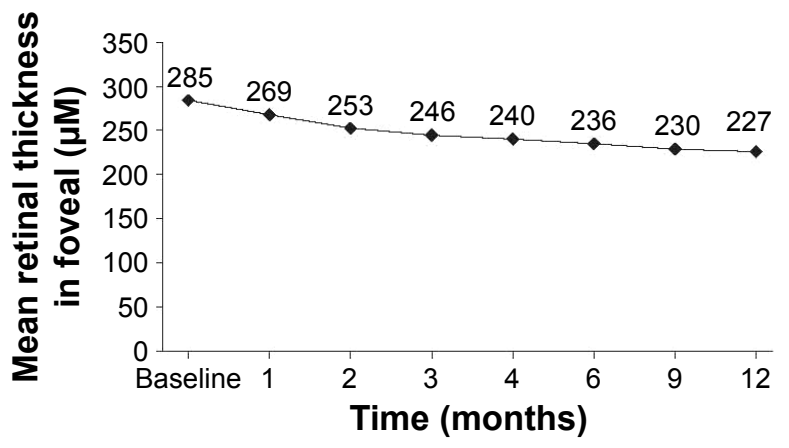

Figure 2 Change in mean retinal thickness after treatment with intravitreal aflibercept for myopic choroidal neovascularization. with laser photocoagulation, Jalkh et $\mathrm{al}^{27}$ found that BCVA improved in only $11 \%$ of eyes, remained unchanged in $21 \%$, and worsened in $68 \%$ (average posttreatment follow-up period 29.2 months).

Although TTT seems to stabilize myopic CNV both clinically and angiographically, with a significant decrease in the activity of lesions, this technique did not result in any significant change in BCVA over a 1-year observation period. ${ }^{7}$ Furthermore, adequate energy levels have not been clearly defined.

PDT with verteporfin was advocated as a safe and welltolerated approach for patients with myopic CNV. Report No 3 of the Verteporfin in Photodynamic Therapy study group is the largest study to analyze the efficacy and safety of PDT as the treatment for subfoveal myopic CNV. At the month 12 examination, $72 \%$ of the verteporfin-treated patients lost fewer than eight letters in BCVA, compared with $44 \%$ of the placebo-treated patients. In addition, $32 \%$ of the verteporfintreated patients versus $15 \%$ of the placebo-treated patients improved by at least five letters. Finally, $86 \%$ of the verteporfintreated patients lost fewer than 15 letters, compared with $67 \%$ of the placebo-treated patients. Results at 2 years were less promising and demonstrated no statistical significance between the eyes treated with PDT and the control groups. ${ }^{6,28}$

Since the advent of intravitreal anti-VEGF agents, it has been possible not only to preserve but also to improve visual acuity in patients suffering from various types of $\mathrm{CNV}$, including myopic $\mathrm{CNV}$.

Many case series on the off-label use of bevacizumab suggest visual improvement with a limited number of injections and an acceptable safety profile. However, there is no highest level of evidence from clinical trials and no regulatory approval for bevacizumab in this indication.

In an open-label study of 17 patients, Arias et $\mathrm{a}^{29}$ found that at the 6-month follow-up, after receiving an average of 1.5 intravitreal bevacizumab injections, mean visual acuity improved by 8.4 letters. A total of $41 \%$ of patients gained at least 1 line of visual acuity, and 17\% gained at least 6 lines. Mean CRT decreased by $79.6 \mu \mathrm{m}$ on OCT. Laud et al ${ }^{30}$ 

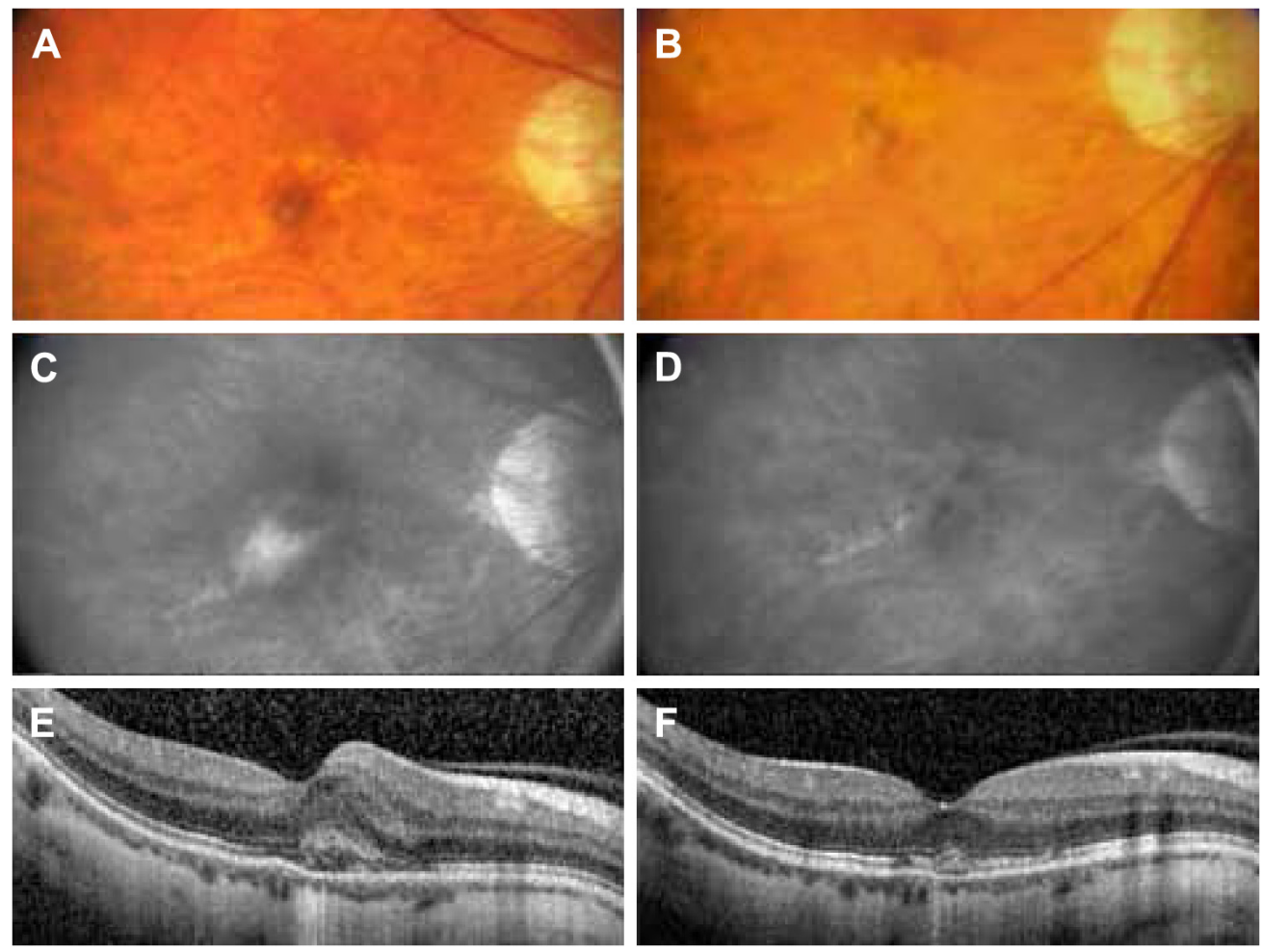

Figure 3 (A) Fundus photograph showing the CNV before treatment. (B) Fundus photograph of the same patient showing CNV after treatment. (C) FA in the late phase (I5 min) showing staining and leakage from the CNV before injection. (D) FA in the late phase (I5 min) showing decreased staining and leakage from the CNV after two injections. (E) OCT image showing increased retinal reflectivity and a hyper-reflective round lesion under the retina corresponding to the CNV before treatment. (F) OCT image after two intravitreal injections showing decreased retinal thickness in foveal.

Abbreviations: CNV, choroidal neovascularization; FA, fluorescein angiography; OCT, optical coherence tomography.

found a mean 1.5 lines improvement in eyes with myopic CNV treated with intravitreal bevacizumab, with a mean follow-up of 7.3 months. In their 24-month study, Baba et $\mathrm{al}^{5}$ showed that intravitreal bevacizumab is more effective than PDT in eyes with myopic CNV. In a case series of 63 eyes with CNV secondary to PM, Ikuno et al ${ }^{31}$ reported improvements in mean BCVA from $0.57 \pm 0.43$ logarithm to the minimal angle of resolution (logMAR) ( 0.27 decimal equivalent) to $0.33 \pm 0.34 \log$ MAR ( 0.47 decimal equivalent) with about one to six bevacizumab injections during the 12 months of observation (mean 2.4 injections). After 12 months, improvement in BCVA of more than 3 lines was noted in 25 (40\%) eyes, worsening of more than 3 lines was observed in three (5\%) eyes, and vision was unchanged in $35(56 \%)$ eyes. Fluorescein leakage from the CNV ceased in 30 (48\%) eyes, diminished in 28 (44\%) eyes, and was unchanged in five $(8 \%)$ eyes.

Ranibizumab has recently received approval in the European Union for the treatment of myopic CNV. This decision was made based on results from the RADIANCE sham-controlled, double-masked randomized clinical trial, which established the superiority of intravitreal ranibizumab over PDT. ${ }^{22}$ RADIANCE and the open-label REPAIR study ${ }^{20,21}$ showed that around $40 \%$ of patients treated with ranibizumab gained 15 or more letters of visual acuity at 3 months, compared with $15 \%$ of PDT-treated patients. Mean visual acuity gain was approximately 14 letters at 1 year after a median of 2.0 ranibizumab injections. ${ }^{20-22}$ A number of small prospective and retrospective studies have reported favorable results with intravitreal ranibizumab for the treatment of myopic CNV. For example, in a series of 16 consecutive patients with myopic $\mathrm{CNV}$, Lai et al ${ }^{32}$ demonstrated a mean improvement in BCVA of 3 lines at 12 months, with 12 (75.0\%) eyes experiencing an improvement of 2 or more lines. A total of 15 (93.8\%) eyes had angiographic CNV closure at 3 months, while two (12.5\%) eyes had recurrence of $\mathrm{CNV}$ and required retreatment between 3 and 9 months. OCT showed a significant reduction in the mean central foveal thickness after treatment with ranibizumab. In another case series, Silva et $\mathrm{al}^{33}$ found that at the 12-month follow-up, the mean visual acuity improved from 20/100 to 20/63 after a mean of 3.6 intravitreal ranibizumab injections. No cases of severe visual acuity loss occurred, and no systemic or ocular side effects were registered during the follow-up. However, of the 32 patients with CNV secondary to PM at baseline, only nine patients completed 6 months of follow-up. 
The results of the MYRROR study of intravitreal aflibercept in myopic CNV were published in 2015. The authors reported a mean change of +13.5 letters in BCVA in aflibercept-treated patients compared with +3.9 letters in patients receiving sham treatment (and receiving aflibercept injections at week 24) and maintenance of gains in visual acuity through week 48. A direct comparison of BCVA results between the current study and MYRROR is not possible given the different ways in which vision was measured (decimal vs Early Treatment Diabetic Research Study chart letters); however, both studies demonstrated significant improvements in BCVA in eyes treated with aflibercept. These two studies reported unidirectional changes of mean CRT in the macular area. The MYRROR study showed significant decreases from $349.7 \mu \mathrm{m}$ at baseline to $263.5 \mu \mathrm{m}$ at week 48 . In our study, CRT decreased significantly from $285 \mu \mathrm{m}$ at baseline to $227 \mu \mathrm{m}$ at month 12 . Special mention should be made of the schedule of injections. In our study, patients received two mandatory loading injections (at baseline and week 4) followed by PRN retreatment. Patients received a mean of 2.6 injections overall, 2.0 injections in the first 4 weeks, and 0.6 injection during the remainder of the study (48 weeks). In the MYRROR study, patients received a single mandatory loading injection followed by PRN retreatment. The median number of injections was 2.0 in the first study quarter and 0 in the remaining quarters.

In most studies of ranibizumab, the mean baseline BCVA was similar (0.2-0.3 in decimal equivalent $)^{22,31-33}$ and in all studies, improvement in mean BCVA of $\geq 2-3$ lines was noted. However, in studies with 12 months of follow-up, there was a difference in the mean number of injections (from one to four). ${ }^{22,31-33}$

The results from the literature regarding ranibizumab and bevacizumab treatment are in accordance with the findings from the current study, which demonstrated that intravitreal aflibercept is a safe and efficacious treatment option in myopic CNV. The gain in visual acuity was swift and was maintained throughout the study. Mean BCVA significantly improved by almost 3 lines in logMAR equivalent in conjunction with decreasing CRT. Patients maintained visual and anatomic improvements after 12 months with an average of 2.6 intravitreal aflibercept injections. The number of injections needed to achieve visual and anatomic improvements is also consistent with the literature about other anti-VEGF drugs in patients with myopic CNV.

The MYRROR study group reported that serious adverse events occurred in seven patients in the intravitreal aflibercept treatment group. Of these, only one ocular serious adverse event (macular hole) was reported. No deaths were reported over the 48-week follow-up period. The most frequently reported adverse events were eye pain (7.7\%) and conjunctival hemorrhage $(11.0 \%) .{ }^{23}$ In the current study, there were no serious adverse events. The rates of eye pain and subconjunctival hemorrhage were similar compared with MYRROR (10\% and 7\%, respectively).

The higher incidence of serious adverse events in the MYRROR study may be related to the larger sample size (four times as many patients as in our study). Most serious adverse events are rare and may not occur in a smaller treatment group such as ours.

Clinical trials of ranibizumab for myopic $\mathrm{CNV}$ (RADIANCE and REPAIR) also demonstrated low rates of serious adverse events and ocular adverse events. ${ }^{21,22}$ In the REPAIR study, one case of culture-negative endophthalmitis considered by investigators to be related to the injection was reported. ${ }^{21}$

\section{Strength and limitations}

The strength of this study is its ability to show the significant visual and anatomical improvements that can be achieved in patients with myopic CNV treated with intravitreal aflibercept in routine clinical practice. The inability to directly compare results with the MYRROR study due to differences in follow-up period and BCVA measurement is a limitation.

\section{Conclusion}

The 12-month results of the current study of intravitreal aflibercept for myopic CNV using a PRN regimen were positive. A total of $27(87 \%)$ eyes experienced improvement of $\geq 1$ line in BCVA, with a gain of $\geq 3$ lines in five $(16 \%)$ eyes. None of the treated eyes lost $\geq 1$ line of vision. Intravitreal aflibercept retreatment guided by disease activity criteria based on OCT and BCVA was able to provide visual acuity benefits without requiring FA. Overall, intravitreal aflibercept was well tolerated in patients with myopic $\mathrm{CNV}$ over 12 months in our study.

\section{Acknowledgments}

The authors have taken full responsibility for the scope, direction, and content of the manuscript and have approved the final manuscript. Medical writing support was provided by Corey Eagan, MPH, of PAREXEL and was funded by Bayer HealthCare Pharmaceuticals.

\section{Disclosure}

The authors report no conflicts of interest in this work. 


\section{References}

1. Wong TY, Ferreira A, Hughes R, Carter G, Mitchell P. Epidemiology and disease burden of pathologic myopia and myopic choroidal neovascularization: an evidence-based systematic review. Am J Ophthalmol. 2014;157(1):9-25.

2. Ohno-Matsui K, Yoshida T, Futagami S, et al. Patchy atrophy and lacquer cracks predispose to the development of choroidal neovascularisation in pathological myopia. Br J Ophthalmol. 2003;87(5):570-573.

3. Wakabayashi T, Ikuno Y. Choroidal filling delay in choroidal neovascularisation due to pathological myopia. Br J Ophthalmol. 2010;94(5): 611-615.

4. Ikuno Y, Jo Y, Hamasaki T, Tano Y. Ocular risk factors for choroidal neovascularization in pathologic myopia. Invest Ophthalmol Vis Sci. 2010;51(7):3721-3725.

5. Baba T, Kubota-Taniai M, Kitahashi M, Okada K, Mitamura Y, Yamamoto S. Two-year comparison of photodynamic therapy and intravitreal bevacizumab for treatment of myopic choroidal neovascularisation. Br J Ophthalmol. 2010;94(7):864-870.

6. Blinder KJ, Blumenkranz MS, Bressler NM, et al. Verteporfin therapy of subfoveal choroidal neovascularization in pathologic myopia: 2-year results of a randomized clinical trial - VIP report no. 3. Ophthalmology. 2003;110(4):667-673.

7. Ozdek S, Hondur A, Gurelik G, Hasanreisoglu B. Transpupillary thermotherapy for myopic choroidal neovascularization: 1-year follow-up: TTT for myopic CNV. Int Ophthalmol. 2005;26(4-5):127-133.

8. Virgili G, Menchini F. Laser photocoagulation for choroidal neovascularisation in pathologic myopia. Cochrane Database Syst Rev. 2005; 19(4):CD004765.

9. Papadopoulos N, Martin J, Ruan Q, et al. Binding and neutralization of vascular endothelial growth factor (VEGF) and related ligands by VEGF Trap, ranibizumab and bevacizumab. Angiogenesis. 2012;15(2): 171-185.

10. Deissler HL, Lang GK, Lang GE. Capacity of aflibercept to counteract VEGF-stimulated abnormal behavior of retinal microvascular endothelial cells. Exp Eye Res. 2014;122:20-31.

11. Rosenfeld PJ, Brown DM, Heier JS, et al. Ranibizumab for neovascular age-related macular degeneration. $N$ Engl J Med. 2006;355(14): 1419-1431.

12. Brown DM, Michels M, Kaiser PK, Heier JS, Sy JP, Ianchulev T. Ranibizumab versus verteporfin photodynamic therapy for neovascular age-related macular degeneration: two-year results of the ANCHOR study. Ophthalmology. 2009;116(1):57-65.

13. Heier JS, Brown DM, Chong V, et al. Intravitreal aflibercept (VEGF trapeye) in wet age-related macular degeneration. Ophthalmology. 2012; 119(12):2537-2548.

14. Brown DM, Campochiaro PA, Singh RP, et al. Ranibizumab for macular edema following central retinal vein occlusion: six-month primary end point results of a phase III study. Ophthalmology. 2010;117(6): 1124-1133.

15. Campochiaro PA, Heier JS, Feiner L, et al. Ranibizumab for macular edema following branch retinal vein occlusion: six-month primary end point results of a phase III study. Ophthalmology. 2010;117(6):1102-1112.

16. Heier JS, Clark WL, Boyer DS, et al. Intravitreal aflibercept injection for macular edema due to central retinal vein occlusion: two-year results from the COPERNICUS study. Ophthalmology. 2014;121(7): $1414-1420$.

Clinical Ophthalmology

\section{Publish your work in this journal}

Clinical Ophthalmology is an international, peer-reviewed journal covering all subspecialties within ophthalmology. Key topics include: Optometry; Visual science; Pharmacology and drug therapy in eye diseases; Basic Sciences; Primary and Secondary eye care; Patient Safety and Quality of Care Improvements. This journal is indexed on Submit your manuscript here: http://www.dovepress.com/clinical-ophthalmology-journal
17. Ogura Y, Roider J, Korobelnik JF, et al. Intravitreal aflibercept for macular edema secondary to central retinal vein occlusion: 18 -month results of the phase 3 GALILEO study. Am J Ophthalmol. 2014;158(5): 1032-1038.

18. Nguyen QD, Brown DM, Marcus DM, et al. Ranibizumab for diabetic macular edema: results from 2 phase III randomized trials: RISE and RIDE. Ophthalmology. 2012;119(4):789-801.

19. Korobelnik JF, Do DV, Schmidt-Erfurth U, et al. Intravitreal aflibercept for diabetic macular edema. Ophthalmology. 2014;121(11): 2247-2254.

20. Tufail A, Patel PJ, Sivaprasad S, et al. Ranibizumab for the treatment of choroidal neovascularisation secondary to pathological myopia: interim analysis of the REPAIR study. Eye (Lond). 2013;27(6):709-715.

21. Tufail A, Narendran N, Patel PJ, et al. Ranibizumab in myopic choroidal neovascularization: the 12-month results from the REPAIR study. Ophthalmology. 2013;120(9):1944-1945.

22. Wolf S, Balciuniene VJ, Laganovska G, et al. RADIANCE: a randomized controlled study of ranibizumab in patients with choroidal neovascularization secondary to pathologic myopia. Ophthalmology. 2014;121(3): 682-692.

23. Ikuno Y, Ohno-Matsui K, Wong TY, et al. Intravitreal aflibercept injection in patients with myopic choroidal neovascularization: the MYRROR Study. Ophthalmology. 2015;122(6):1220-1227.

24. Macular Photocoagulation Study Group. Laser photocoagulation of subfoveal neovascular lesions of age-related macular degeneration. Updated findings from two clinical trials. Arch Ophthalmol. 1993;111(9): 1200-1209.

25. Macular Photocoagulation Study Group. Visual outcome after laser photocoagulation for subfoveal choroidal neovascularization secondary to age-related macular degeneration. The influence of initial lesion size and initial visual acuity. Arch Ophthalmol. 1994;112(4):480-488.

26. Secretan M, Kuhn D, Soubrane G, Coscas G. Long-term visual outcome of choroidal neovascularization in pathologic myopia: natural history and laser treatment. Eur J Ophthalmol. 1997;7(4):307-316.

27. Jalkh AE, Weiter JJ, Trempe CL, Pruett RC, Schepens CL. Choroidal neovascularization in degenerative myopia: role of laser photocoagulation. Ophthalmic Surg. 1987;18(10):721-725.

28. Verteporfin in Photodynamic Therapy Study Group. Photodynamic therapy of subfoveal choroidal neovascularization in pathologic myopia with verteporfin. 1-year results of a randomized clinical trial - VIP report no 1. Ophthalmology. 2001;108(5):841-852.

29. Arias L, Planas N, Prades S, et al. Intravitreal bevacizumab (Avastin) for choroidal neovascularisation secondary to pathological myopia: 6-month results. Br J Ophthalmol. 2008;92(8):1035-1039.

30. Laud K, Spaide RF, Freund KB, Slakter J, Klancnik JM Jr. Treatment of choroidal neovascularization in pathologic myopia with intravitreal bevacizumab. Retina. 2006;26(8):960-963.

31. Ikuno Y, Sayanagi K, Soga K, et al. Intravitreal bevacizumab for choroidal neovascularization attributable to pathological myopia: one-year results. Am J Ophthalmol. 2009;147(1):94-100.

32. Lai TY, Chan WM, Liu DT, Lam DS. Intravitreal ranibizumab for the primary treatment of choroidal neovascularization secondary to pathologic myopia. Retina. 2009;29(6):750-756.

33. Silva RM, Ruiz-Moreno JM, Rosa $P$, et al. Intravitreal ranibizumab for myopic choroidal neovascularization: 12-month results. Retina. 2010; 30(3):407-412.

PubMed Central and CAS, and is the official journal of The Society of Clinical Ophthalmology (SCO). The manuscript management system is completely online and includes a very quick and fair peer-review system, which is all easy to use. Visit http://www.dovepress.com testimonials.php to read real quotes from published authors. 